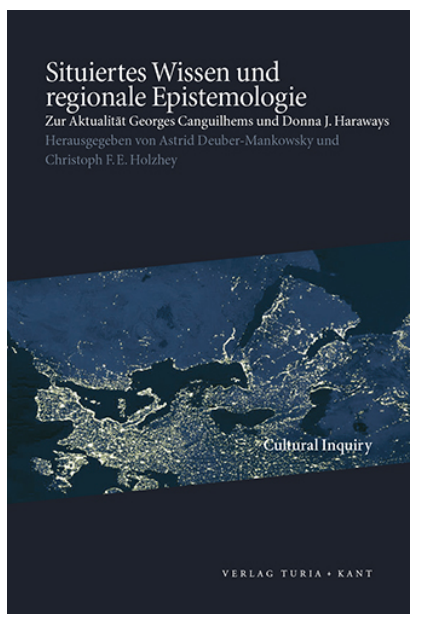

Situiertes Wissen und regionale Epistemologie: Zur Aktualität Georges Canguilhems und Donna J. Haraways, ed. by Astrid Deuber-Mankowsky and Christoph F. E. Holzhey, Cultural Inquiry, 7 (Wien: Turia + Kant, 2013), pp. 87-104

\section{FRANÇOISE BALIBAR}

\section{Von Canguilhem zu Haraway}

CITE AS:

| Françoise Balibar, »Von Canguilhem zu Haraway«, in Situiertes Wissen und regionale Epistemologie: Zur Aktualität Georges Canguilhems und Donna J. Haraways, hg. v. Astrid Deuber-Mankowsky und Christoph F. E. Holzhey, Cultural Inquiry, 7 (Wien: Turia + Kant, 2013), S. 87-104 <https://doi.org/10.37050/ci-07_04>

\section{RIGHTS STATEMENT:}

(C) by the author(s)

This version is licensed under a Creative Commons AttributionShareAlike 4.0 International License. 


\section{VON CANGUILHEM ZU HARAWAY}

Françoise Balibar

1. »Die Objektivität ist der Syndikalismus der Erkenntnis «, so Georges Canguilhem. Gewiss, er formulierte dies nicht öffentlich, sondern auf einem losen Blatt, auf dem er allem Anschein nach einige Ideen zu Papier brachte zeitgleich mit einem auf vier weiteren Blättern, detailliert und sorgfältig verfassten Plan für ein Seminar mit dem Titel »Wie ist die Beziehung von Objektivität und Objekt aufzufassen? «. ${ }^{1}$ Dieses Seminar war Teil der Lehrveranstaltung, die Canguilhem im Universitätsjahr 1958-59 den Studierenden der Sorbonne anbot. Dass das Blatt, auf dem nichts weiter als jener recht provokante Satz steht, in den Ordner mit dem Seminarplan zu Objekt und Objektivität hineingerutscht ist, so als ob es nachträglich hinzugefügt worden wäre, lässt vermuten, dass Canguilhem wahrscheinlich nicht die Absicht hatte, diesen Aphorismus ex cathedra zum Besten zu geben, sondern sich den Entschluss dazu je nach Verlauf des Seminars vorbehalten wollte.

Wie dem auch sei - dieser Satz steht so da, schwarz auf weiß. Als ich ihn las, so muss ich zugeben, war ich ratlos. Objektivität gleichzusetzen mit »einer Bewegung zur Vertretung der Interessen einer Berufskategorie « - was für eine bizarre Metapher (vorausgesetzt, es ist überhaupt eine)! Was ist damit gemeint? Dass es die Funktion der Objektivität ist, die Interessen der Erkenntnis zu vertreten - wem und was gegenüber? Gegen die stets drohende Subjektivität? Oder ist darunter vielmehr zu verstehen, dass die Objektivität die Interessen derjenigen verteidigt, die Erkenntnis hervorbringen? Dass sie letztendlich nur eine Form von korporatistischer Forderung ist - eine Anspielung also auf den angeblich Objektivität begründenden Konsens (der weitestgehend durch institutionelle Machtbeziehungen verfälscht ist), mit dem sich die Gelehrtengemeinschaft brüstet und bewusst davon absieht, dass dieser Konsens sich auf die definitive Ausschließung von Nicht-Gelehrten stützt, ein Umstand, der uns nicht gleichgültig lassen dürfe? - Es scheint sich also um ein bekanntes Lied zu handeln, mit dem uns die Sociology of Scientific Knowledge (SSK), auch »Konstruktivismus « genannt, seit über einem Vierteljahrhundert in den Ohren liegt. »Objectivity is social «, so David Boor, der damit in drei Worten die Essenz seiner The- 
orie zusammenfasst. ${ }^{2}$ "Jedes Wissen ist ein verdichteter Knoten in einem agonistischen Machtfeld. [...] Wissenschaft ist ein anfechtbarer Text und ein Machtfeld [...] «, schreibt Donna J. Haraway und fügt hinzu, diese Logorrhö mache sie »nervös «. ${ }^{3}$ Die Wissenschaft ist ein soziales Konstrukt, keine unvoreingenommene Tätigkeit (wer hat jemals daran geglaubt?), vielmehr ein Unternehmen im Dienste partikularer Interessen, eine Institution der Macht usw. usw. Canguilhem - ein Konstruktivist ohne es zu wissen?

Sehr bald wurde mir klar, dass ich einer falschen Fährte gefolgt war. Zunächst steht das Wort Syndikalismus erst seit kurzem, sagen wir seit den siebziger Jahren, unter dem Verdacht, eine korporatistische Verteidigung der Interessen bestimmter Kategorien zu bezeichnen. In den Jahren 1958-59 war der Syndikalismus eine Bewegung, die in erster Linie auf die Vereinigung der Arbeiter abzielte - und erst in zweiter Linie auf die Verteidigung ihrer Interessen; eine Verteidigung, die erst durch die Vereinigung möglich gemacht wurde. Von Vorrang war aber die Vereinigung der Arbeiter. Indes ist die »Vereinigung der Arbeiter der Beweisführung [l'union des travailleurs de la preuve] « ein Ausdruck, dessen unsterblicher Urheber Gaston Bachelard ist, ${ }^{4}$ der seinerseits von Victor Hugo inspiriert wurde, so sehr drängt sich die Analogie zu dessen Arbeitern des Meeres auf. Mit Sicherheit dachte Canguilhem an diese Formulierung Bachelards, die er später noch kommentieren sollte, als er 1958-59 die Metapher (wenn es denn eine ist) vom Syndikalismus der Erkenntnis hinschrieb: Der Syndikalismus ist die Vereinigung der Arbeiter der Beweisführung und die Objektivität der Syndikalismus der Erkenntnis.

Der Zweifel am metaphorischen Charakter des Ausdrucks rührt daher, dass Canguilhem wie Bachelard unablässig auf der Vorstellung von Arbeit, ja von Schufterei insistiert (eine ziemlich unpassende Formulierung, doch warum nicht die "Zwangsarbeiter der Beweisführung ", wenn man schon dabei ist?). »Die Vereinigung der Arbeiter der Beweisführung «, schreibt Canguilhem 1963, »so lautet die bewundernswerte Formulierung, durch die Bachelard uns lehrt, dass Wissenschaft gemacht wird, und zwar nicht nur dadurch, dass man zusammen an einem Beweis arbeitet, sondern dass man zusammen den Beweis bearbeitet. " ${ }^{5}$ Und er zeigt auf, wie ein solches Programm, indem es zugleich eine mathematische Theorie und eine Experimentaltechnik der Kausalität beinhaltet, »die menschliche Vereinnahmung der Natur besiegelt «. ${ }^{6}$ 
2. Offensichtlich unterscheiden sich Canguilhem und Haraway hierin in radikaler Weise, denn Haraway weist bekanntermaßen jegliche Form des Übergriffs auf die Natur zurück. Dies zu behaupten, heißt die Möglichkeit zu unterstellen, dass sich beide davor nicht hätten unterscheiden können. Denn um sich von einem bestimmten Augenblick an zu unterscheiden und von da an getrennte Wege zu gehen, muss man im Vorfeld den Weg ein Stück weit zusammen beschritten haben. Und ich denke, dass genau dies im Falle von Canguilhem und Haraway zutrifft. Innerhalb eines jeweils verschiedenen Horizonts formuliert, gründen die Überlegungen des einen wie der anderen auf der Feststellung der Unzulänglichkeit der Wissenschaft, sobald sie es mit dem Leben zu tun hat.

Weder der eine noch die andere ist an demjenigen Typ von Unzulänglichkeit interessiert, den Wissenschaftler mit dem angelsächsischen Begriff bias bezeichnen und woraus die Wissenschaftssoziologen ihr Kapital bezogen haben. Donna Haraways Feststellung der Unzulänglichkeit der Wissenschaften rührt aus ihrer Praxis als Forscherin in einem Labor für Entwicklungsbiologie, aus der ihr erstes Buch entstanden ist, das die Geschichte der Embryologie in der Zwischenkriegszeit erzählt, die von der Konfrontation zwischen organizistischen und reduktionistischen Auffassungen (bezüglich des Verhältnisses der Biologie zur Physik) gekennzeichnet ist. ${ }^{7}$ Diese Erfahrung führte sie zu einem Sinneswandel, den man als epigenetisch bezeichnen mag, ${ }^{8}$ aber auch zur Überzeugung - in der sie durch ihr feministisches Engagement noch bestärkt wurde -, dass »the old view of progressive, control-oriented, objective descriptions of nature « inadäquat sei. ${ }^{9}$ Georges Canguilhem lastet die Unzulänglichkeit der Wissenschaft (in seiner medizinischen Doktorarbeit Essai sur quelques problèmes concernant le normal et le pathologique von 1943) zunächst einem Vergessen der Technik zugunsten der Wissenschaft an, das einsetzt, sobald es sich ums Leben handelt; und da ihm zufolge das Leben besonders enge Verbindungen mit der Technik (vgl. die Automaten) unterhält, wird durch eben dieses Vergessen die Wissenschaft vom Leben fern gehalten und im Bereich des Lebendigen unwirksam. 20 Jahre später kommt Canguilhem, stark beeindruckt von den Konzepten der genetischen Biologie, ${ }^{10}$ noch einmal auf seine Position zu sprechen. Der Irrtum, das Kernstück der genetischen Theorie, erscheint ihm nun als dasjenige, was das Leben und die Wissenschaft gemeinsam haben, denn »es gibt [...] keinen Unterschied zwischen dem Irrtum des Lebens und dem des Denkens ", ${ }^{11}$ was ihn wiederum dazu zwingt, seine Auffassung über die Beziehungen von Leben 
und Wissenschaft zu berichtigen: Die Wissenschaft hält nicht vom Leben fern, an dem sie teilhat. Sie ist nur ein Irrtum des Lebens, ${ }^{12}$ sie gehört ihm an - was die 1943 entwickelte Argumentation über den jeweiligen Ort der Wissenschaft und der Philosophie in keinster Weise entkräftet, sondern eher noch stützt. Die Wissenschaft bringt Gesetze hervor - ein Ausdruck dessen, was Canguilhem wissenschaftliche Wahrheit nennt -, und die Philosophie legt die Normen wissenschaftlicher Wahrheit fest. Als kritische Instanz der Infragestellung aller Funktionen des Lebens, die sie nicht einfach als "gegeben « akzeptiert - weder die wissenschaftlichen Äußerungen innewohnenden Wahrheitswerte noch jedwedes andere Produkt menschlicher Tätigkeit -, bewertet die Philosophie den Wert der Normen wissenschaftlicher Wahrheit. Die Rolle des Wissenschaftsphilosophen im Besonderen besteht nun darin festzustellen, an welcher normativen Wahl diese oder jene Äußerung teilhat, und eine Bewertung dieser vorzunehmen. In Bezug auf die Wissenschaft ist die Philosophie demnach nicht ohne Objekt - anders als die positivistische Vulgata, die spontan von der Mehrzahl der Forscher übernommen wird, glauben machen will. An dieser Stelle kann eines der berühmtesten Zitate Canguilhems in Erinnerung gerufen werden: »Es gibt keine Wahrheit außer der wissenschaftlichen [...]. Aber zu sagen, es gibt keine Wahrheit außer der wissenschaftlichen oder keine Objektivität außerhalb der wissenschaftlichen Erkenntnis, soll dennoch nicht heißen, dass die Philosophie keinen Gegenstand hat. «13

Nun ist auch dasjenige, was in Donna Haraways Arbeiten über Wissenschaft und wissenschaftliche Objektivität besonders besticht, die völlig unerwartete und aus positivistischem Blickwinkel unwillkommene Einführung der Frage nach den Werten in die Wissenschaft. Was sie im Blick hat, ist:

das Projekt einer Nachfolgewissenschaft [...], das eine adäquatere, bessere und reichere Darstellung [account] einer Welt, in der ein gutes Leben möglich sein soll, anbietet, und das ein kritisch-reflexives Verhältnis zu unseren eigenen wie auch zu fremden Herrschaftspraktiken und dem für jede Position konstitutiven, unterschiedlichen $\mathrm{Ma}$ an Privilegiertheit und Unterdrückung ermöglicht. ${ }^{14}$

Sie fügt mit Blick auf traditionelle Unterscheidungen hinzu, dass es sich hierbei um ein Problem handele, dass eher in den Bereich der Ethik (die Moral) und Politik als den der Epistemologie fällt. Doch ist es nicht gerade ein Markenzeichen des Werks Canguilhems, sich durch philoso- 
phische Reflexion in die Politik einzuschalten, ohne jemals das Wort "politisch « zu gebrauchen? ${ }^{15}$ Und ist nicht der Satz Haraways, den ich gerade zitiert habe, ein Satz von Canguilhem, in jene besondere Sprache übersetzt, die dem Englischen verwandt, aber so eigentümlich für Haraway ist? Eine Vielzahl von Zitaten ließe sich in diesem Sinne anführen, ich beschränke mich aber auf jenes, in dem Haraway ihr Problem, » > unser Problem «, definiert, das darin besteht,

wie wir zugleich die grundlegende historische Kontingenz aller Wissensansprüche und Wissenssubjekte in Rechnung [account] stellen, eine kritische Praxis zur Wahrnehmung unser eigenen bedeutungserzeugenden, ssemiotischen Technologien entwickeln und einem nicht-sinnlosen Engagement für Darstellungen [accounts] verpflichtet sein können, die einer 'wirklichen< Welt die Treue halten, einer Welt, die teilweise miteinander geteilt werden kann $[\ldots] .{ }^{16}$

Abgesehen davon, dass hier in aller Deutlichkeit gesagt wird, dass es nicht darum geht, den Anspruch aufzugeben, Rechenschaft (account) über die Welt (rreak world) in einer sinnhaften Form abzulegen (eine Möglichkeit, die die SSK von vornherein ablehnt), denke ich, dass vor allem die Nähe dieser Aussage zu bestimmten Sätzen Canguilhems festzuhalten ist, in denen dieser Émile Meyersons Ansatz kritisiert, Erkenntnis als Übereinstimmung von Realität und Quantität zu definieren: »Gleichwohl darf man nicht vergessen, daß die wissenschaftliche Erkenntnis zwar Qualitäten als imaginär darstellt und so für ungültig erklärt, sie aber deswegen doch keineswegs annullieren kann. Die Quantität ist die negierte, nicht jedoch die liquidierte Qualität. « ${ }^{17}$

3. Nachdem ich nun herausgestellt habe, inwiefern Haraway und Canguilhem eine bestimmte allgemeine Orientierung teilen, möchte ich auf das kommen, was sie trennt. Das Wort »Arbeit « konnte hierfür bereits einen Hinweis liefern - ein Wort, das von Canguilhem immer wieder aufgegriffen wird und das man bei Haraway eher mit der Lupe suchen muss, mit Ausnahme einiger recht zweideutiger, dem Marxismus gewidmeter Passagen, etwa in dem Text mit dem Titel »Animal Sociology and a Natural Economy of the Body Politic: A Political Physiology of Dominance ${ }^{18}$ Diese unterschiedliche Gewichtung von Arbeit lässt sich nicht einfach mit den unterschiedlichen Epochen ihres Schaffens begründen: Canguilhem als jemand, der in einer von der marxistischen Lehre durchdrungenen Welt lebt, gegenüber Haraway, die in einer Epoche lebt, in 
der man sich nicht einmal das Wort zu äußern traut, da das von ihm Bezeichnete so vielen Individuen abgeht. ${ }^{19}$ Ich glaube, dass die Differenz zwischen beiden grundlegender ist und in ihren gegensätzlichen Positionen zum Ort des Menschen in der Natur begründet liegt. Der Gebrauch, den der eine und die andere vom Wort »Objektivität « machen, und die Bedeutungen, die beide ihm zubilligen, tragen die Züge dieser Differenz.

In den bereits erwähnten Seminarnotizen beginnt Canguilhem in guter französischer Professorenmanier zunächst damit, eine Unterscheidung zwischen zwei Auffassungen von Objektivität einzuführen, je nachdem sie vom Objekt ausgeht oder zu ihm hinführt. Und er eröffnet den ersten Teil seines Seminars (»Vom Objekt zur Objektivität «) auf folgende Weise: »Erkenntnis ist eine Spielart der Passivität des Gemüts. Es kommt nun ganz und gar darauf an, innerhalb dieser Passivität die Passivität der Kontemplation (Idee) von der Passivität der Passion (Idole) zu unterscheiden. " Die Wortwahl ist ein Gemisch aus kartesischen (Passivität des Gemüts, Kontemplation, Passion) und baconschen (Idole, Idee) Zutaten. Dabei ist daran zu erinnern, dass Canguilhem sich an Philosophiestudierende Ende der 1950er Jahre wendet (nur 10\% eines Jahrgangs hatten das Baccalauréat und damit die Studienzugangsberechtigung), die versiert waren in solchen begrifflichen Unterscheidungen, wie sie die Geschichte der Philosophie hervorbringt: "Mit Descartes beginnen « drängte sich geradezu auf.

Es ist bemerkenswert, dass es bei dieser Herangehensweise "ganz und gar darauf an[kommt] «, die Spreu vom Weizen zu trennen und die Fehlerquellen zu beseitigen (die von Bacon verzeichneten und klassifizierten Idole). Denn in dem Moment, da sich eine Erkenntnis herausbildet, empfängt das Gemüt - kraft seiner passiven Natur selbst oder gewissermaßen seiner selbst zum Trotz - die Idole und Ideen (nach Bacon), die Wahrheit und den Irrtum (nach Descartes) in völlig ungeordneter Form und nur die (der Wahrheit angemessene) Kontemplation erlaubt es, das eine vom anderen zu trennen, und damit auch, dem Impuls der Passion zu widerstehen (»Objektiv sein heißt, einem Impuls widerstehen «, notiert Canguilhem). Daher stellten im 17. Jahrhundert Descartes und Bacon, die gleichwohl nicht die gleiche Auffassung von Wissenschaft vertraten, ein ganzes Arsenal von Bedingungen auf (die Trockenheit des Auges, die das optische Instrument bei Bacon erfüllt; die Schlichtheit der mathematischen Gleichung bei Descartes), die es erlauben, die Idee in ihrer Reinheit zu isolieren, sie gewisser- 
maßen vom Scheidewerk zu befreien und einem Erzklumpen gleich zu »behandeln «, aus dem man Metall zu gewinnen versucht.

All dies jedoch unter der Bedingung, dass dies möglich ist, was jedoch nie ganz der Fall ist, denn hinter dem Instrument gibt es immer ein menschliches und daher feuchtes Auge und auch die analytische Geometrie hat ihre Grenzen... In rascher Weise (zumindest den Notizen zufolge, im mündlichen Vortrag wohl ausführlicher) zeichnet Canguilhem die bedeutendsten Etappen im Prozess der Herstellung einer wissenschaftlichen Tatsache aus einer rohen Tatsache (der Ausdruck stammt von Poincaré $)^{20}$ nach - eine echte Arbeit, die all das umfasst, was im Wortfeld von Transformation mitschwingt. Zu Recht sieht Canguilhem in der von Newton veranlassten Einführung des Kalküls in die Physik eine entscheidende Etappe der Transformationsarbeit eines irgendwie als »natürlich « wahrgenommenen zu einem künstlichen Objekt, einem letztlich unentwirrbaren Geflecht aus Beobachtung und Kalkül: Was Keppler (mit bloßem Auge oder mithilfe seines Fernrohrs) am Himmel »zu lesen glaubte", wird von Newton abgeleitet, herausgearbeitet, hergestellt und mit mathematischen Symbolen geschrieben, auf Grundlage der Axiome, die seine drei Bewegungsgesetze darstellen. »Dieses Mal«, so Canguilhem, »ist die Erkenntnis keine Passivität mehr. «

Ich denke nicht, dass Donna Haraway diesen ersten Teil von Canguilhems Seminar ablehnen würde, beschreibt er doch einen gewissen historischen Prozess, von dem sich schwer behaupten lässt, dass er nicht stattgefunden habe. Canguilhem und sie weichen jedoch in der Interpretation der Frage voneinander ab, wie man sich angesichts dieses Stands der Dinge zu verhalten habe. Während Canguilhem, nachdem er festgestellt hat, dass man von der Passivität zur Aktivität des Gemüts (d. h. des Subjekts, um mit Kant zu sprechen) übergegangen sei, mit dem zweiten Teil seines Seminars fortfährt (»Von der Objektivität zum Objekt « - ich werde später noch darauf zurückkommen), wirft Haraway ihrerseits ein: Halt! Lasst uns hier nicht noch weiter gehen, lasst uns den Rückzug antreten! Lasst uns ein Manöver vornehmen (»ein vermeintlich einfaches Manöver ", so schreibt sie), ${ }^{21}$ das bereits durch die (hegelsche?) Dialektik in Gang, aber nicht zum Abschluss gebracht wurde. Lasst uns damit aufhören, das Objekt der Erkenntnis als passives Objekt anzusehen, das man wie Bodenschätze behandelt, das man transformiert, indem man es wie Brotteig bearbeitet, das man symbolisch repräsentiert, nachdem man es von seinem Aufputz frei 
gemacht hat. Lasst es uns als Akteur und Agenten betrachten - »an actor and an agent « im Original, eine dieser unübersetzbaren Stellen. Das Wort "agency", das in der klassischen englischen Philosophie gebraucht wird, um eine allgemeine und unzureichend definierte Eigenschaft des Handelns zu bezeichnen (Hume merkt an, die Worte efficacity, agency, power, force, energy, necessity, connection und productive quality seien in einem solchen Maße synonym, dass es absurd sei, das eine von ihnen zur Erklärung eines anderen zu verwenden), ${ }^{22}$ hat im Zuge seiner Verwendung bei angelsächsischen Philosophen des 20. Jahrhunderts, insbesondere bei John Austin und Stanley Cavell, heutzutage eine Bedeutung angenommen, in der die Unterscheidung aktiv/passiv verwischt und der Bereich der Ungewissheit ausgeleuchtet wird, die über den Ursachen und Ursprüngen der eigentlichen Handlungen schwebt (was im Übrigen dem Sprachgebrauch des Englischen entspricht, in dem das Passiv geläufiger ist als im Französischen). Daher nimmt es nicht Wunder, dass Donna Haraway, diese Expertin im Zerschlagen von Dichotomien, dieses Wort, das letztlich weder Fisch noch Fleisch ist, gebraucht und - mit bemerkenswerter Feinheit in der Beherrschung unterschiedlicher Bedeutungsnuancen - sogar so weit geht, von der "active agency « der Welt zu sprechen, um dem Status des Objekts in der Welt gewissermaßen einen neuen Look zu verpassen.

4. Wenn ich von »neuem Look « spreche, tue ich dies mit voller Absicht. Denn genau darum geht es: Die Welt, so Haraway, hat ihren Look, ihr Aussehen, verändert; die Garderobe der Metaphern, die sie einhüllen und womit wir sie einkleiden, wurde erneuert. Letztendlich passen die bislang verwendeten Metaphern nicht mehr zur analogen und somit figurativen Bezeichnung der konzeptuellen Entitäten, die sie beschreiben. Bereits in ihrem ersten Buch Crystals, Fabrics and Fields. Metaphors That Shape Embryos stellte sich Donna Haraway die Aufgabe, die Stichhaltigkeit der Begriffe zu analysieren, die, aus der Umgangssprache übernommen und daher zwangsläufig metaphorisch, von der Wissenschaft verwendet werden, um die Dinge der Welt zu benennen. Doch ist die Welt eben nicht aus »Dingen «, aus perfekt abzugrenzenden Objekten gemacht. Bereits in ihrem ersten Buch ließ sie Descartes (wie jeder weiß, der Anwalt der klaren und distinkten Vorstellungen) den Kopf hinhalten, indem sie ihn für die Illusion verantwortlich machte, die darin besteht, die Welt als eine aus klaren und distinkten Dingen gemachte $\mathrm{zu}$ betrachten. ${ }^{23}$ Wie könnte also dasjenige, was nicht einmal 
mehr zur Beschreibung der Objekte der klassischen Physik geeignet ist (neben den Teilchen, den Gegenständen par excellence, mussten Objekte anderer Natur eingeführt werden, etwa Felder von unbegrenzter Ausdehnungen, die sich im Laufe der Zeit andersartig im Raum entwickeln), Aufschluss geben über die »Objekte « der Biologie? Die Welt der Biologie erscheint eher als eine Abfolge hierarchisch geordneter Ebenen, doch erst am Ende unzähliger Verzögerungen und abgebrochener Versuche findet die Biologie in ihrer jeweiligen Entwicklungsetappe die ihr angemessene, stets vorläufige Metapher - in der von Haraway in ihrem ersten Buch von 1976 untersuchten Epoche stellt diese der Organismus dar. In »Situiertes Wissen « kommt sie auf diesen Punkt zurück, wobei sie ihre Überlegungen noch zuspitzt. Die Welt, so Haraway, ist nicht in klaren und distinkten Vorstellungen zu erfassen. Ebenso wenig ist sie ein großes Buch, das darauf wartet, von jenen gelesen zu werden, die im Besitz des Kodes sind (Folgt meinem Blick!). Vielmehr ist sie von Agenten gemacht, ja, sogar von Kojoten, jenem Tier, das Haraway auswählt, um es zum Emblem zu machen. Denn, so erklärt sie, die Figur des Kojoten, dieses als besonders listig geltenden Tiers, entspricht in der indianischen Mythologie dem Fuchs der europäischen Fabeln. Er ist ein Tier, das Menschen immer wieder ins Gehege kommt (ein veritabler Gegenstand*), stets dort auftaucht, wo man ihn nicht erwartet, und ist dermaßen verschlagen, dass er kaum zu fassen ist. Nur schwerlich lässt sich ermitteln, ob Haraway damit zu provozieren sucht (ich stelle mir das Gesicht der Physiker vor, denen man sagt, dass sie in ihren Berechnungen und Versuchsanordnungen die kojotische Natur der Elektronen in Betracht ziehen müssen, allein schon, weil sie weder Wellen noch Teilchen sind) oder ob sie tatsächlich glaubt, was sie schreibt (eigentlich ist das, was sie hier auf humoristische Weise vorschlägt, die völlig neue Ausweitung einer in ihrer Ernsthaftigkeit kaum zu überbietenden empiristischen Doktrin). Wahrscheinlich ist beides der Fall.

5. Jedenfalls sind wir mit der Einführung des Kojote-Objekts in die Objektivitätsfrage recht weit entfernt von der kantischen Auffassung und der sich daraus ergebenden Konstituierung des wissenschaftlichen Objekts, so wie sie Canguilhem seinen Studierenden im Jahr 1958-59 darlegt. Ich war bei Canguilhems Satz »Dieses Mal ist die Erkenntnis keine Passivität mehr « stehengeblieben und hatte festgestellt, dass sich von dort an die Wege Haraways und Canguilhems unweigerlich trennen mussten. Tatsächlich ist der darauffolgende Satz Canguilhems von 
Haraways Standpunkt aus inakzeptabel: »Von hier nimmt eine neue Theorie des wissenschaftlichen Objekts ihren Anfang, die von einer Objektivität ausgeht, die als eine gewisse konstituierende Aktivität des Objekts verstanden wird « - eines Objekts, so Canguilhem, dessen Passivität nicht eigens präzisiert werden muss, sondern bei dem es zu unterstreichen gilt, dass es erst durch Aufrufung des Subjekts konstituiert wird, demgegenüber es nicht die Position eines Gegenstands* wie das vormalige Objekt der Wissenschaft, sondern eher die Position eines »Projekts « einnimmt. Sich an ein in der Philosophiegeschichte geschultes Publikum richtend, gibt Canguilhem daraufhin zu bedenken, dass »sich diese Theorie der konstituierenden Objektivität nicht völlig des Bezugs auf das Objekt entledigen kann«:

[Kant] bleibt dem Verhältnis zwischen der Erkenntnis und dem Objekt der Wahrnehmung verpflichtet. Selbst wenn er die Wahrnehmung auf die Wissenschaft ausrichtet, begreift er die Wissenschaft als Wahrnehmung. Denn Begriffe ohne Anschauung sind leer. Es wird also weiterhin ein Objekt beibehalten, das man nicht erkennen kann, obwohl man es denken kann. ${ }^{24}$

In diesem Sinne stellt das wissenschaftliche Objekt (Canguilhem unterstreicht das Wort), das Objekt der Wissenschaft, kein Ob-jekt, sondern ein Pro-jekt dar.

Nichts von alledem bei Haraway, wo der Begriff des Objekts, eines grundlegend empirischen Objekts, darauf abzielt, über die Welt Rechenschaft abzulegen (»account for « ist das von ihr mit Nachdruck gebrauchte Wort). Während für Canguilhem die wissenschaftliche Objektivität (für Canguilhem eine redundante Ausdrucksweise, da er keine andere Objektivität als die wissenschaftliche in Betracht zieht) darauf abzielt, mit einer der Mathematik eigenen Gewissheit einen winzigen Ausschnitt der Welt zu erkennen (nämlich jenen, den der Filter der Objektivität zurückbehält), versucht Haraway ihrerseits nichts beiseite zu lassen und so viel wie möglich zu umfassen. Während die objektive Wissenschaft die Qualität des Wissens und die mit ihr verbundene Gewissheit privilegiert (denn erst die Objektivität ermöglicht, wie man weiß, die Mathematisierung) und dabei eine Einengung ihres Tätigkeitsbereichs in Kauf nimmt, versucht Haraway im Gegensatz dazu, sich von den durch die Sorge um Objektivität erforderlichen Grenzziehungen zu lösen und nimmt dabei in Kauf, nicht mehr als »accounts « einfordern zu können; bei »account « klingt zwangsläufig auch »count« mit, das 
Rechnen, das Kalkül, die schlicht numerischen Teile der Mathematik, an denen das Universum im Übrigen sehr reich ist.

Ebenso wenig wie die Verfechter einer objektiven Wissenschaft trauert Haraway dem nach, worauf sie verzichtet - in ihrem Fall die Leistungsfähigkeit der Mathematik, im Fall Ersterer die Fähigkeit, die Welt als Ganze zu erfassen. Vielmehr rühmen sich beide ihres Verzichts: So wie die Physik sich damit tröstet, ein unpräzises Konzept (die »Realität «) durch ein anderes von mathematischer Natur ersetzt zu haben (die "physikalische Realität ", deren Gewissheit und Präzision nicht angefochten werden können), so begrüßt auch Haraway die - unbestreitbare - sprachliche Überlegenheit von Erzählungen (der literarischen Form der »accounts «) über wissenschaftliche Äußerungen.

6. Ausgehend von dieser schematischen Gegenüberstellung Haraways und Canguilhems komme ich nun auf die Objektivität zurück, und zwar die wahre, wissenschaftliche, so wie sie Canguilhem seinen Studierenden gegenüber darlegt. Ich komme deshalb darauf zurück, weil ich der Auffassung bin, dass Donna Haraway in ihrem Wunsch, den partikularen Wissensformen (situated knowledges) einen Wert zurückzuerstatten, den fast vier Jahrhunderte »objektiver Wissenschaft " praktisch annulliert haben, sich eine Vorstellung von wissenschaftlicher Objektivität macht, die nicht exakt ist. Ich meine damit nicht etwa falsch, sondern unvollständig und folglich inexakt. Ihre Auffassung entspricht genauer jener, die sich "praktizierende « Wissenschaftler von ihrer Tätigkeit machen, und die sich, um es kurz zu machen, als "positivistisch « bezeichnen lässt, insofern sie dem nachdrücklich betonten Wunsch folgt, nichts mit philosophischem Denken zu tun haben zu wollen. Und genau an dieser Stelle drängt sich Canguilhems Analyse der Rolle der Philosophie als einer kritischen Instanz der Bewertung von wissenschaftlichen Wahrheitsnormen und von Objektivität auf.

Wie Lorraine Daston in ihrem 2007 erschienenen, zusammen mit Peter Galison verfassten Buch Objectivity bemerkt (in dem es ihr irgendwie gelingt, Haraway nicht einmal zu zitieren), taucht das Wort »Objektivität « in der wissenschaftlichen Literatur erst Mitte des 19. Jahrhunderts auf ${ }^{25}$ - zu einem relativ späten Zeitpunkt, an dem sich im Übrigen jener Umsturz vollzieht, auf den Canguilhem sein Seminar aufbaut (der Übergang von der »Objektivität mit Objekt « zur »Objektivität ohne Objekt«). Seit ungefähr 1850 verwendet die Physik (und nur sie, insbesondere die theoretische Physik, was natürlich ein nicht zu 
unterschätzender Umstand ist) den Begriff einer Objektivität ohne Ontologie, und zeitgleich hält auch das Wort »Objektivität ", bis dahin philosophisch konnotiert, Einzug in die Sprache der Physiker (und in deren Gefolge auch anderer Wissenschaftler).

In Anbetracht dieses neuen, den Physikern eigenen Gebrauchs des Wortes »Objektivität « möchte ich in aller Kürze daran erinnern, dass das abstrakte Verständnis von Objektivität die Grundlage schlechthin der Physik darstellt, auch wenn das Wort im 18. Jahrhundert noch nicht existierte und die Physik selbst erst im 19. Jahrhundert sich dessen gewahr wurde, was sie seit ihrer Entstehung der Objektivität zu verdanken hatte. Ich bin sogar der Auffassung, dass die Vorstellung der Objektivität bereits in dem Relativitätsprinzip am Werk ist - das man nach Galilei benannt hat, obwohl er es nie geäußert hatte -, ja, dass sie sogar dessen Wesen darstellt. Grundsätzlich fordert dieses Prinzip, in der Vielfalt von Phänomenen die Gleichwertigkeit (und nicht etwa die Identität) bestimmter Gesichtspunkte (oder »Situationen ", wie Haraway, so scheint es mir, sagen würde) zu ermitteln. Diese Gesichtspunkte ergeben nicht dieselbe Sichtweise auf die Welt (mir ist bewusst, dass es sich hier um einen metaphorischen Gebrauch des Wortes "Sicht" handelt), doch sind sie gleichwertig in dem Sinne, dass die Unterschiede zwischen den Sichten, die sie freigeben, verblassen, sobald berücksichtigt wird, dass die »Punkte ", von denen aus diese »Sichten « »eingenommen « werden, unterschiedlich sind - der Beweis dafür, dass sich hinter diesen unterschiedlichen Sichtweisen etwas »Reales « befindet, das für alle gleich ist (»gleich « diesmal im Sinne von identisch), eine Welt, die wir »mit anderen denkenden Wesen gemein haben « und die demnach »objektiv « ${ }^{26} \mathrm{im}$ banalen Sinne des Wortes ist (man kann sich über die "Realität " dessen, was gesehen wird, verständigen). Die Gleichwertigkeit der Gesichtspunkte ist dasjenige, was die Identität des Objekts konstruiert.

Entscheidend ist dabei der Umstand, dass diese Gleichwertigkeit an Bedingungen geknüpft ist: Nicht alle Gesichtspunkte (alle »Situationen «) sind untereinander gleichwertig; zwei »Situationen « sind nur dann gleichwertig, wenn sie sich in einem bestimmten Bewegungszustand auf einer Gerade und mit gleicher Geschwindigkeit unterscheiden. Von geringer Bedeutung ist dabei die Form, die die Bedingung jeweils einnimmt; wichtig ist allein, dass die Gleichwertigkeit der Gesichtspunkte überhaupt einer Bedingung unterworfen ist. In diesem Sinne stellt die Physik einen speziellen Relativismus dar: Nicht alle Gesichtspunkte sind gleichwertig, doch gibt es eine ganze Klasse gleichwertiger 
Gesichtspunkte, d. h. solche, die in ihrer jeweiligen Beschreibung der Phänomene gleich viel wert sind und von denen weder einer besser als der andere, noch einer von ihnen zu privilegieren ist.

Aus diesem Grund erzürnt es mich, wenn ich bei Haraway (und mehr noch bei anderen) lese, dass die Physik eine einheitliche Sichtweise hervorbringe, eine Sichtweise, die von nirgendwo erfolgt (»der Blick von nirgendwo «), von niemanden ausgeht, incognito, ${ }^{27}$ die Sichtweise Gottes (»der göttliche Trick «) usw. usw. ${ }^{28}$ Das ist falsch. Zwischen dem Relativismus (alle Gesichtspunkte sind gleichwertig) und dem Absolutismus (ein einziger Gesichtspunkt ist legitim) existiert eine Auffassung, die sich weder auf das eine noch aufs andere beschränkt, nämlich die Auffassung der wissenschaftlichen Objektivität (genauer noch: im Sinne der Physik), derzufolge der individuelle Gesichtspunkt keinen Wert an sich besitzt, sondern nur insofern, als er von anderen geteilt wird (was einen Teil seines Inkognitos lüftet und Gott von der Sache ausschließt), die der Zahl nach unbegrenzt sind und eine Klasse gleichwertiger "Beobachter « bilden - gleichwertig in ihrer jeweiligen Beschreibung der Welt.

Die Verkennung dieser Vorstellungen, die die Physik begründet haben und dies auch weiterhin tun, hätte kaum Bedeutung (man kann ja nicht alles wissen), wenn sie nicht eine solche nicht zu unterschätzende Rolle in Donna Haraways Argumentation spielen würde. Haraway glaubt in der Objektivität, die mit Anspruch auf einen einheitlichen (und daher universalistischen, göttlichen usw.) Gesichtspunkt gleichgesetzt wird, den Ursprung dessen gefunden zu haben, was nicht geleugnet werden könne, denn schließlich sei es eine historische Tatsache: Die objektive Wissenschaft habe an allen Beherrschungsunternehmungen des Westen teilgenommen, die Unterwerfung der Frau mit eingerechnet. Von hier ist es nur ein kleiner Schritt dazu, ihr einen dominierenden Charakter zuzuweisen. Indem die Argumentationskette, die gerade ausgerollt wurde, wieder zurückgerollt wird, kann dann dieser Herrschaftscharakter auf seinen Ursprung - den Objektivitätsanspruch - zurückgeführt werden. Haraway zögert nicht, von einem Prinzip zu sprechen: »the principle of domination is deeply imbedded in our natural sciences $\ll .{ }^{29}$

7. Beim Wiederlesen meiner Ausführungen stelle ich fest, dass sie unverkennbar engagiert (»situated «) sind. Ich erhebe mir selbst gegenüber den sich aufdrängenden Einwand, der lautet: Zu groß scheint mein persönliches Interesse an der Objektivität, so wie sie sich in unserem Jahr- 
hundert darstellt, um unparteiisch zu sein. Wahrscheinlich ist das so. Wenn ich mich frage, worin eigentlich die Nachteile des Objektivitätsanspruchs bestehen, fällt mir sofort eine Antwort ein: Objektivität ist fruchtbar im Sinne einer sterilen Fruchtbarkeit. Fruchtbar, das ist nicht weiter der Rede wert, doch eine Fruchtbarkeit, die eben steril ist, weil sie es nicht erlaubt, den Bereich der physikalischen Mathematik, ihren Herkunftsort, zu verlassen. Sie ist nicht einmal fähig, Zugänge zum Bereich der Biologie zu eröffnen. Mehr als nur gewisse Beobachtungstechniken (Laser, Bilder aller Art etc.) und eventuell einige analog funktionierende Modelle importieren zu wollen, ist purer Nonsens, eine reduktionistische Halluzination der Physiker. Denn diese technischen Importe machen aus der Biologie noch keine Erweiterung der Physik. (Das ist, als ob man aus Lavoisiers Einsatz von Tauben zum Nachweis von Sauerstoff in seinen Gläsern - siehe das berühmte Bild von David schlussfolgern würde, er betreibe Biologie.) Die Konzepte der Physik bringen im Bereich des Lebendigen nichts hervor - das ist von vornherein klar und mittlerweile bewiesen.

Aber ist das wirklich so klar? Woher kommt es, dass sich die Physik auf unbelebte Objekte beschränkt hat? Handelt es sich um eine freiwillige Entscheidung oder hat sich eher die Gewohnheit durchgesetzt, ohne dass man darauf Acht gegeben hat? Immerhin hatte Galilei keine Angst davor, seine Beweisführungen auf Beispiele zu stützen, die dem Bereich des Lebendigen entlehnt waren (der Flug der Vögel, der Lauf eines Pferdes), ebenso wie er dem Aufkeimen des Kerns eines Orangenbaums aus China mehr Bedeutung als seiner Haltbarkeit beimaß. ${ }^{30}$ Sicherlich sind diese Andeutungen nur flüchtig und zielen nicht darauf ab, das Leben zu erklären, aber zumindest behandeln sie die Welt des Lebendigen nicht als etwas nicht zur Sache Gehöriges. Explizit ist dies bei Newton der Fall, der ebenso gut wie Descartes für die Sackgasse, in der wir uns befinden, verantwortlich sein könnte. Gleich nachdem er sein drittes Gesetz anführt, jenes, das die Verwendung des Paares Aktion/Reaktion in der Physik definiert, schreibt Newton: »If a horse draws a stone tied to a rope, the horse (if I may say so) will be equally drawn back towards the stone. ${ }^{31}$ Dies ist das erste Mal, dass ein Pferd als Lebewesen (es zieht einen Stein) reduziert wird auf einen durch einen Koeffizienten (seinen Schwerpunkt und seine Masse) zugewiesenen Punkt; und Newton bittet durch jenes »If I may so « um Verzeihung, aus einem Lebewesen ein unbelebtes Objekt gemacht zu haben. Sehr bald sollte es aber nicht mehr nötig sein, um Verzeihung zu bitten: So besei- 
tigt die Marquise von Châtelet in ihrer Übersetzung ganz einfach diesen Passus, der sie offenbar stört. ${ }^{32}$ Und auf diese Weise gerät man in die von Haraway beschriebene Sackgasse, wonach heute ein im Krankenhaus, besonders nah am Lebendigen arbeitender Biologe in wenig überschwänglicher, aber für mich anregender Weise verlautbart: »Das Problem in der Biologie ist, dass es keinen Gegenstand gibt. « In dieser Hinsicht scheint mir Donna Haraways Antwort kaum befriedigend: Kann man sich tatsächlich mit "accounts « der Welt zufrieden geben, mit Ausdrucksformen der »situated knowledges «, die sicherlich nichts und niemanden vergessen (und darin der »realen « Welt treuer sind als die objektive Wissenschaft), aber dennoch keine Erkenntnis darstellen, da sie ohne Beziehung zueinander sind, eine Art Kaleidoskop, »eine Vielheit von bestimmten wirklichen Einzelwesen « ${ }^{33}$

Ich möchte nur schematisch ein weiteres Argument anführen, von dem ich denke, dass es Donna Haraway überzeugen müsste, die wissenschaftliche Objektivität nicht über Bord zu werfen. Die Kategorie der »Beziehungen « spielt bei ihr eine wichtige Rolle: Ihre Welt ist eine Welt der Beziehungen. Und gerade die logische Kategorie der Gleichwertigkeit (die sich, so viel ist klar, in Anbetracht der Gesichtspunkte oder »Situationen " gemäß dem Objektivitätsanspruch an die Stelle der Kategorie der Identität setzen sollte), impliziert, dass das Definierte (das Objekt, über dessen »Realität " sich die Repräsentanten verschiedener gleichwertiger Gesichtspunkte verständigen, das »Objekt « im Sinne des von der Objektivität Anvisierten, das Projekt also) nicht etwa, was eine allzu nahe liegende Vorstellung ist, eine Sammlung isolierter Tatsachen ist, sondern vielmehr die Beziehungen zwischen diesen Tatsachen darstellt. Kurz gesagt, identisch sind für alle gleichwertigen Gesichtspunkte nicht die Dinge, sondern die Beziehungen unter ihnen. Wie Poincaré schreibt:

Die Wissenschaft ist [...] ein System der Beziehungen [...]. [N]ur in den Beziehungen muß die Objektivität gesucht werden [...]. Die äußeren Gegenstände zum Beispiel, für die das Wort Objekt erfunden worden ist, sind eben Objekte und nicht flüchtige und ungreifbare Erscheinungen, weil es nicht nur Gruppen von Empfindungen sind, sondern Gruppen, die durch eine beständige Verbindung zusammengekittet sind. Diese Verbindung ist es, und nur sie, was an ihnen Objekt ist, und dies ist eine Beziehung. ${ }^{34}$ 
Das, woran ich auf den zurückliegenden Seiten erinnert habe, würde ausreichen, um die Äußerung zu bestätigen: Erst die Objektivität stellt das Objekt der Physik her (Canguilhem notiert seinerseits: »Die Objektivität ist eine Konzeption, das heißt eine Generierung « [Hervorhebung F.B.]). Doch ist es bemerkenswert, dass es durch eine Verallgemeinerung der Objektivität zu einem alle Formen von Invarianz umfassenden Begriff (inklusive derjenigen, die nicht nur die Position des Objekts in Raum und Zeit, sondern auch seine interne Struktur betreffen) möglich ist aufzuzeigen, dass die elementaren Objekte der Physik (»Teilchen « heißt es manchmal, obwohl die Metapher völlig unpassend ist) durch ihre Eigenschaften der Invarianz definiert sind. Daher ist es nicht falsch zu behaupten, dass die elementaren Objekte der Teilchenphysik durch eine solch erweiterte Objektivität "generiert « werden (oder genauer noch: durch die Anforderungen einer solchen Objektivität in einem weiteren Sinne).

Aus dem Französischen von Konrad Petrovszky

\section{A N M ER K U N G E N}

1 Die Archive Georges Canguilhems werden im Centre d'Archives de Philosophie, d'Histoire et d'Edition des Sciences (CNRS / ENS Ulm) aufbewahrt.

2 David Bloor, "A Sociological Theory of Objectivity «, Royal Institute of Philosophy Lecture Series, 17 (1984), S. 229-45.

3 Donna J. Haraway, "Situiertes Wissen. Die Wissenschaftsfrage im Feminismus und das Privileg einer partialen Perspektive", in dies., Die Neuerfindung der Natur. Primaten, Cyborgs und die Frauen, hg. v. Carmen Hammer u. Immanuel Stieß (Frankfurt a.M.: Campus, 1995), S. 73-97, hier S. 75.

4 Siehe etwa Gaston Bachelard, Le rationalisme appliqué (Paris: PUF, 1949), dessen drittes Kapitel den Titel »Rationalisme et corrationalisme, l'union des travailleurs de la preuve« trägt.

5 Georges Canguilhem, »Gaston Bachelard et les philosophes «, zuerst erschienen in Sciences, 24 (1963), S. 7-10, wieder abgedruckt in ders., Études d'histoire et de philosophie des sciences, 7. erw. Aufl. (Paris: Vrin, 1994), S. 187-95, hier S. 192.

6 Von Gaston Bachelard, L'activité rationaliste de la physique contemporaine (Paris: PUF, 1951), S. 218 entliehene Formulierung.

7 Donna J. Haraway, Crystals, Fabrics, and Fields. Metaphors That Shape Embryos (Berkeley: North Atlantic Books, 2004).

8 Gegenwärtig "werden als epigenetisch jene biologischen Phänomene bezeichnet, die nicht auf rigide Art und Weise durch die genetische Hülle bedingt sind « (Alain Prochiantz, Les stratégies de l'embryon. Embryons, gènes, évolution [Paris: PUF, 1988], S. 155). Im Allgemeinen stellt die Epigenese eine Lehre dar, 
derzufolge die Entwicklung einer Zelle (oder des gesamten Organismus) nicht allein durch interne Mechanismen (heutzutage der genetische Code), sondern in gleicher Weise durch die Interaktion zwischen dem Organismus und seiner Umwelt reguliert wird.

9 Haraway, Crystals, S. 2.

101961 legten Jacques Monod, François Jacob und André Lwoff die Idee eines genetischen Programms vor.

11 Georges Canguilhem, Das Normale und das Pathologische, übers. v. Monika Noll u. Rolf Schubert (München: Hanser, 1974), S. 194.

12 Siehe dazu Guillaume Le Blanc, Canguilhem et les normes (Paris: PUF, 1998), S. $107 \mathrm{ff}$.

13 So Canguilhem anlässlich einer von der Zeitschrift Revue présente der »Union rationaliste" organisierten Diskussion am 27. Februar 1968, veröffentlicht in Structuralisme et marxisme, hg. v. Jean-Marie Auzias u. a. (Paris: Union Générale d'Edition, 1970), S. 205-65, hier S. 249.

14 Haraway, "Situiertes Wissen «, S. 78.

15 Muss an dieser Stelle noch daran erinnert werden, dass Canguilhem der Politik als Mitglied der Resistance seine Jugendjahre gewidmet hat?

16 Haraway, »Situiertes Wissen «, S. 78.

17 Canguilhem, Das Normale, S. 71.

18 In Donna J. Haraway, Simians, Cyborgs and Women. The Reinvention of Nature (London: Routledge, 1991), S. 7-20. Haraway wird im Allgemeinen als Sozialistin im amerikanischen Sinn des Worts, d. h. als Marxistin, dargestellt, was sie aber nicht daran hindert, oft sehr kritisch zu sein. So kommt es vor, dass sie marxistische Thesen (etwa: »The labour process constitutes the fundamental human condition «, ebd., S. 10) in solch ironischer Weise vorbringt, dass man sich fragt, ob gerade sie oder die Vulgata spricht.

19 Tatsächlich spricht Donna Haraway von Arbeit oder genauer: von neuen Arbeitsformen im Cyborg-Manifest (siehe den Abschnitt »Hausarbeitsökonomie« in »Ein Manifest für Cyborgs. Feminismus im Streit mit den Technowissenschaften ", in dies., Die Neuerfindung, S. 54-59) als Organisationsstruktur des globalen Kapitalismus neueren Typs, die die marxistische Analyse nicht zu beschreiben vermag.

20 Vgl. Henri Poincaré, Der Wert der Wissenschaft (Leipzig: Teubner, 1910), S. 165-76.

21 Haraway, »Situiertes Wissen «, S. 93.

22 Vgl. David Hume, A Treatise of Human Nature, hg. v. L. A. Selby-Bigge u. P. H. Nidditch (Oxford: Clarendon Press, 1975), Book I, Part 3, Section XIV. Vgl. auch den Eintrag "Agency in Vocabulaire Européen des Philosophies, hg. v. Barbara Cassin (Paris: Seuil/Robert, 2004), S. 26-32.

23 Haraway, Crystals, S. 10. Es wäre ein Leichtes, den amerikanischen Feminismus dadurch zu karikieren, dass man einige Aussagen isoliert und aufbauscht, die aus Descartes den Vorsänger der Herrschaft des Mannes über die Natur und somit zum Erzfeind machen.

24 Wiederum aus Canguilhems unveröffentlichten Seminar »Wie ist die Beziehung 
von Objektivität und Objekt aufzufassen? «, das er 1958-59 an der Sorbonne abhielt. (Übersetzung von Konrad Petrovszky.)

25 Lorraine Daston und Peter Galison, Objektivität (Frankfurt a.M.: Suhrkamp, 2007), S. 32.

26 Vgl. Poincaré, Der Wert der Wissenschaft, S. 197. Fällt ein Stein vom Mast eines Schiffes, sieht ein Beobachter auf dem Kai etwas anderes als einer auf dem Schiff: Für den einen beschreibt der Stein eine Kurve (eine Parabel), für den anderen eine gerade vertikale Linie. Die Relativitätstheorie besagt indes, dass beide Beobachter sich über die Tatsache verständigen können, dass sie ungeachtet ihrer unterschiedlichen Situation (am Kai stehend ist der eine unbeweglich, während der andere durch eine gewisse Geschwindigkeit des Schiffes vorangetrieben wird) dasselbe Phänomen, den Fall des Steines gesehen haben, wenn auch aus unterschiedlichen Winkeln, was auch die unterschiedlich Beschreibung dessen, was sie gesehen haben, erklärt.

27 Tatsächlich schreibt Baudelaire: »Der Beobachter ist ein Fürst, der überall sein Inkognito genießt. ( ( Der Maler des modernen Lebens «, in Charles Baudelaire, Aufsätze zur Literatur und Kunst 1857-1860, übers. v. Friedhelm Kemp u. Bruno Streiff [München: Hanser, 1989], S. 213-58, hier S. 222).

28 Haraway, »Situiertes Wissen «, S. 80-81.

29 Haraway, "Animal Sociology «, S. 8.

30 Galileo Galilei, Dialog über die beiden hauptsächlichsten Weltsysteme, das ptolemäische und das kopernikanische, übers. v. Emil Strauss (Darmstadt: Wissenschaftliche Buchgesellschaft, 1982), z. B. S. 193-97 und S. 221-22.

31 Das dritte Newton-Axiom lautet: »To every action is always opposed a certain reaction, or, the mutual actions of two bodies upon each other are always equal, and directed to contrary parts. " (Isaac Newton, Mathematical Principles of Natural Philosophy, 2 Bde., hg. v. Florian Cajori [New York: Greenwood Press, 1962], I, S. 13). Es sei daran erinnert, dass das Datum der Ersterscheinung der Principia Mathematica 1687 ist.

32 Zur Abtrennung des Lebendigen durch die Physik zum Zeitpunkt der wissenschaftlichen Revolution, einem kaum behandelten Thema, siehe Jean Starobinski, Aktion und Reaktion. Leben und Abenteuer eines Begriffspaars, übers. v. Horst Günther (Frankfurt a.M.: Suhrkamp, 2003).

33 Alfred North Whitehead, Prozeß und Realität. Entwurf einer Kosmologie, übers. v. Hans-Günter Holl (Frankfurt a.M.: Suhrkamp, 1979), S. 130.

34 Poincaré, Der Wert der Wissenschaft, S. 200. 
Françoise Balibar, »Von Canguilhem zu Haraway«, in Situiertes Wissen und regionale Epistemologie: Zur Aktualität Georges Canguilhems und Donna J. Haraways, hg. v. Astrid Deuber-Mankowsky und Christoph F. E. Holzhey, Cultural Inquiry, 7 (Wien: Turia + Kant, 2013), S. 87-104 <https://doi.org/10.37050/ci-07_04>

\section{REFERENCES}

Auzias, Jean-Marie (Hg.), Structuralisme et marxisme (Paris: Union Générale d'Edition, 1970)

Bachelard, Gaston, L'activité rationaliste de la physique contemporaine (Paris: PUF, 1951)

Le rationalisme appliqué (Paris: PUF, 1949)

Baudelaire, Charles, Aufsätze zur Literatur und Kunst 1857-1860, übers. v. Friedhelm Kemp u. Bruno Streiff (München: Hanser, 1989)

Bloor, David, $\gg$ A Sociological Theory of Objectivity «, Royal Institute of Philosophy Lecture Series, 17 (1984), S. 229-45 <https://doi.org/10.1017/S0957042X00002005>

Canguilhem, Georges, Das Normale und das Pathologische, übers. v. Monika Noll u. Rolf Schubert (München: Hanser, 1974)

$\gg$ Gaston Bachelard et les philosophes «, Sciences, 24 (1963), S. 7-10

Études d'histoire et de philosophie des sciences, 7. erw. Aufl. (Paris: Vrin, 1994)

Cassin, Barbara (Hg.), Vocabulaire Européen des Philosophies (Paris: Seuil/Robert, 2004)

Daston, Lorraine u. Peter Galison, Objektivität (Frankfurt a.M.: Suhrkamp, 2007)

Galilei, Galileo, Dialog über die beiden hauptsächlichsten Weltsysteme, das ptolemäische und das kopernikanische, übers. v. Emil Strauss (Darmstadt: Wissenschaftliche Buchgesellschaft, 1982)

Haraway, Donna J., Crystals, Fabrics, and Fields. Metaphors That Shape Embryos (Berkeley, CA: North Atlantic Books, 2004)

Simians, Cyborgs and Women. The Reinvention of Nature (London: Routledge, 1991)

— $\gg$ Situiertes Wissen. Die Wissenschaftsfrage im Feminismus und das Privileg einer partialen Perspektive «, in dies., Die Neuerfindung der Natur. Primaten, Cyborgs und Frauen, hg. u. eingel. v. Carmen Hammer u. Immanuel Stieß (Frankfurt a.M.: Campus, 1995), S. 73-97

Hume, David, A Treatise of Human Nature, hg. v. L. A. Selby-Bigge u. P. H. Nidditch (Oxford: Clarendon Press, 1975)

Newton, Isaac, Mathematical Principles of Natural Philosophy, 2 Bde., hg. v. Florian Cajori (New York: Greenwood Press, 1962)

Poincaré, Henri, Der Wert der Wissenschaft (Leipzig: Teubner: 1910)

Prochiantz, Alain, Les stratégies de l'embryon. Embryons, gènes, évolution (Paris: PUF, 1988)

Starobinski, Jean, Aktion und Reaktion. Leben und Abenteuer eines Begriffspaars, übers. v. Horst Günther (Frankfurt a.M.: Suhrkamp, 2003)

Whitehead, Alfred North, Prozeß und Realität.Entwurfeiner Kosmologie, übers. v. Hans-Günter Holl (Frankfurt a.M.: Suhrkamp, 1979) 